\title{
Relação público-privada na política brasileira de atenção cardiovascular de alta complexidade
}

\author{
Petter Ricardo de Oliveira 1 \\ Mariana Guerra 2 \\ Adalmir de Oliveira Gomes ${ }^{3}$ \\ Aiane Luiz Martins ${ }^{4}$ \\ 1 Universidade de Brasília, Brasília/DF - Brasil \\ 2 Universidade de Brasília / Programa de Pós-Graduação em Contabilidade, Brasília / DF - Brasil \\ 3 Universidade de Brasília / Programa de Pós-Graduação em Administração, Brasília / DF - Brasil \\ ${ }^{4}$ Instituto Euro Americano de Educação, Ciência e Tecnologia, Brasília / DF - Brasil
}

A saúde especializada no Brasil é oferecida pelo setor privado sob regulamentação e financiamento públicos desde a década de 1950, mantendo-se após a promulgação da Constituição Federal de 1988, quando foi criado o Sistema Único de Saúde (SUS). Nas últimas décadas a participação do setor privado aumentou no atendimento terciário, inclusive nos serviços cardiovasculares, gerando mudanças no SUS. Este estudo analisa a relação público-privada na Política Nacional de Atenção Cardiovascular de Alta Complexidade de 2008 a 2014. Os resultados indicam que, comparativamente ao setor público, o setor privado responde por uma maior participação tanto no atendimento terciário para condições cardiovasculares quanto no recebimento de recursos para o fornecimento de serviços de saúde. Isso aponta para uma contradição na gestão do sistema de saúde no Brasil, que, apesar de público, universal e livre, tem privilegiado o setor privado.

Palavras-chave: serviço cardiovascular; cuidado terciário; políticas públicas; relações público-privadas.

\section{Relación público-privada en la política brasileña de atención cardiovascular de alta complejidad}

La salud especializada en Brasil es ofrecida por el sector privado bajo regulación y financiamiento públicos desde la década de 1950, y se mantuvo después de la promulgación de la Constitución Federal de 1988, cuando se creó el Sistema Único de Salud (SUS). En las últimas décadas, la participación del sector privado aumentó en la atención terciaria, incluso en los servicios cardiovasculares, generando cambios en el SUS. Este estudio analiza la relación público-privada en la Política Nacional de Atención Cardiovascular de Alta Complejidad de 2008 a 2014. Los resultados indican que, en comparación con el sector público, el sector privado tiene una mayor participación tanto en la atención terciaria para condiciones cardiovasculares como en la recepción de recursos para prestación de servicios de salud. Esto indica una contradicción en la gestión del sistema de salud en Brasil, pues, a pesar de ser público, universal y libre en su concepción, privilegia al sector privado.

Palabras clave: servicio cardiovascular; cuidado terciario; política públicas; relación público-privada.

\section{Public-private relation in the Brazilian policy of tertiary care for cardiovascular conditions}

Specialized health care in Brazil has been provided by the private sector under public regulation and financing since the 1950s. It continued after the promulgation of the 1988 Federal Constitution, which also created the Unified Health System (SUS). In the last decades, the share of the private sector has increased in tertiary care, including cardiovascular services, generating changes in SUS. This study analyzes the public-private relationship in the National Tertiary Care Policy for Cardiovascular Conditions from 2008 to 2014. The results indicate that, compared to the public sector, the private sector has a greater share in both tertiary care for cardiovascular conditions and receivables for providing health services. This points to a contradiction in the management of the health system in Brazil, which, albeit public, all-population-oriented, and free in its conception, has privileged the private sector.

Keywords: cardiovascular service; tertiary care; public policy; public-private relationship. 


\section{INTRODUÇÃO}

As doenças crônicas não transmissíveis são hoje o principal problema de saúde da população mundial. No Brasil, estima-se que $73 \%$ das mortes são causadas por essas doenças, sendo as mais prevalentes as cardiovasculares (30\%), as neoplasias (16\%), as doenças respiratórias crônicas (6\%) e a diabetes mellitus (5\%) (Malta et al., 2014).

O sistema de saúde pública do país organizou redes de atenção à saúde cardiovascular por meio de ações de promoção da saúde, prevenção dos agravos e assistência àqueles que necessitam de intervenções de média e alta complexidade (Mendes, 2011). Entretanto, o setor de saúde pública, historicamente incapaz de atender a todas as demandas, tem cada vez mais recorrido às instituições privadas para prestar serviços de saúde à população brasileira (Romano \& Scatena, 2014). Desde a década de 1950, e mesmo após a promulgação da Constituição Federal de 1988, que marca o surgimento do Sistema Único de Saúde (SUS), a atenção especializada tem sido ofertada, contraditoriamente, pelo setor privado sob regulação e financiamento públicos (Solla \& Chioro, 2014). Essa contradição demanda um conhecimento mais aprofundado da relação público-privada entre o Estado e os prestadores privados de serviços de saúde. ${ }^{1}$

O presente estudo investiga a Política Nacional de Atenção Cardiovascular de Alta Complexidade (PNACAC) a fim de compreender essa relação. Realizou-se um estudo empírico a partir de dados secundários de 2008 a 2014 obtidos de bancos de dados oficiais do SUS (DATASUS). Uma das premissas deste estudo é a de que os interesses dos atores públicos e privados podem ser diferentes e, por vezes, até concorrentes: enquanto o Estado brasileiro tem como princípio orientador a garantia do interesse público (Alexandrino \& Paulo, 2013), o setor privado, em regra, visa ao lucro financeiro. Além disso, deve-se dar especial atenção à relação entre o Estado e as organizações privadas a fim de garantir a universalidade e a integralidade da assistência à saúde no sistema brasileiro.

Alguns estudos (Romano \& Scatena, 2014; Solla \& Chioro, 2014) apontam os efeitos do aumento da participação privada na prestação de cuidados à saúde de alta complexidade no Brasil. Por exemplo, Romano e Scatena (2014) destacam como principais vantagens dessa participação o aumento da rapidez e da capacidade de solução de problemas, a expansão da rede de serviços, a redução dos tempos de espera para cirurgias e a melhoria dos resultados na recuperação dos pacientes. Em contrapartida, Solla e Chioro (2014) sublinham os problemas relativos ao favorecimento que as instituições privadas dão a procedimentos de alto custo.

A prestação de serviços de saúde tem sido estendida para o setor privado no âmbito de um sistema que, na verdade, deveria oferecer atenção integral e universal, tendo o Estado como tutor dos interesses públicos e repressor da demanda. Em outras palavras, o governo pode contratar o setor privado para fornecer serviços que o próprio Estado não consegue suprir, mas este não pode terceirizar a prestação de serviços para atender a toda a demanda, por mais que a demanda por saúde pública seja maior que a oferta do próprio Estado, uma vez que a Constituição Federal de 1988 estabelece um Sistema Único de Saúde público, universal e integral. Sendo assim, a saúde é uma área que precisa de forte intervenção tanto da sociedade quanto do Estado (Gadelha, Quental, \& Fialho, 2003; Gadelha, 2012; Ribeiro, Moreira, Ouverney, \& Silva, 2017).

\footnotetext{
${ }^{1}$ A relação público-privada é um sistema de habilitação, repasse de recursos financeiros e regulação exercido pelos entes públicos (União,
} estados, Distrito Federal e municípios) sobre os prestadores privados de serviços hospitalares de média e alta complexidade. 
Segundo Mello et al. (2017), mais do que um simples caso de coexistência, a interdependência entre Estado e mercado é uma característica comum do SUS. É consenso que o gestor público é incapaz de regular o setor privado por ele contratado, cuja grande influência é reflexo de sua capacidade instalada. No entanto, segundo os referidos autores, não há um padrão homogêneo para todo o país, havendo regiões mais ou menos dependentes do setor privado do que outras. Parece faltar à literatura um estudo descritivo e longitudinal que trate desse padrão no que diz respeito à atenção à saúde de alta complexidade, em geral, e à atenção cardiovascular, em particular (Marchildon, 2015; Oliver, Lorenc, \& Innvær, 2014).

Este estudo tem por foco a prestação de serviços de saúde e parte do pressuposto de que as necessidades de saúde não podem ser previstas uma vez que são irregulares (Arrow, 1963; Grossman, 1972). A prestação de serviços é, no geral, insuficiente para atender a toda a demanda, mas também há casos em que é maior do que o necessário. Outrossim, este estudo tem por foco a dimensão organizacional presente no Modelo de Giovanella e Fleury (1995) e discute a prestação como uma forma de se garantir acesso dentro dos limites geográficos de cada território social (Assis \& Jesus, 2012; Thiede \& McIntyre, 2008).

\section{REVISÃO DA LITERATURA}

\subsection{A Política Nacional de Atenção Cardiovascular de Alta Complexidade (PNACAC)}

De acordo com as diretrizes do SUS, a Política Nacional de Atenção Cardiovascular de Alta Complexidade (PNACAC) visa garantir os princípios da saúde pública no país. Um desses princípios é a universalidade, ou seja, toda a população deve ter acesso a todos os serviços de saúde, o que inclui a atenção integral e especializada para doenças cardiovasculares. Os serviços devem incluir ações humanizadas para prevenção de doenças, promoção da saúde e reabilitação, bem como procedimentos diagnósticos e cirúrgicos. Outro princípio é a descentralização: a política regionaliza os serviços segundo a distribuição demográfica, sendo as redes de saúde estabelecidas de acordo com as necessidades da população (Pinto, Fraga, \& Freitas, 2012; Ribeiro et al., 2017).

A PNACAC organiza hierarquicamente a atenção cardiovascular especializada em redes planejadas em conjunto pelos departamentos ou secretarias de saúde nos estados e nos municípios. Tais redes de atenção consistem em arranjos organizacionais de ações e serviços de saúde com diferentes densidades tecnológicas e estabelecidos para garantir atenção integral por meio de sistemas técnicos, logísticos e de apoio à gestão (Portaria GM/MS n. 4.279, 2010). A PNACAC organiza o setor em seis tipos de serviços, com o respectivo quantitativo mínimo de produção anual: cirurgias cardiovasculares (180 procedimentos); procedimentos de cardiologia intervencionista (144); cirurgias cardiovasculares pediátricas (120); cirurgias vasculares (90); procedimentos endovasculares extracardíacos (120); e laboratório de eletrofisiologia (39).

A referida política também estabelece os requisitos para habilitação dos prestadores de atenção de alta complexidade para condições cardiovasculares. Tais requisitos, contudo, não se baseiam nas demandas regionais/locais, mas sim na capacidade instalada e no número de procedimentos (Portaria SAS/MS n. 433, 2012), o que tem levado à concentração de prestadores de atenção de alta complexidade em grandes centros humanos (Solla \& Chioro, 2014). Assim, a atenção primária tem sido disponibilizada à população de forma "quase universal", mas o encaminhamento aos centros 
de atenção especializada tem sido inadequado, a despeito dos princípios de descentralização e integralidade (Barata, Tanaka, \& Mendes, 2014). Neste contexto, é fundamental investigar os efeitos dos vieses para o setor privado presentes nas políticas públicas de atenção especializada. Como apontam Ribeiro e Alcoforado (2016), deve-se avaliar, em cada caso, as vantagens da governança e dos acordos na implementação de políticas.

\subsection{Financiamento da Atenção de Alta Complexidade no SUS}

A atenção de alta complexidade soluciona aproximadamente $5 \%$ dos problemas de saúde da população, mas constitui o nível de atenção mais caro no âmbito do sistema público de saúde brasileiro, consumindo quase 50\% dos recursos do Ministério da Saúde (Servo, Piola, Paiva, \& Ribeiro, 2011). A razão para os custos elevados está nas próprias características da atenção de alta complexidade, a qual faz uso intensivo de tecnologia e requer profissionais altamente especializados (Solla \& Chioro, 2014).

A atenção de alta complexidade tem sido oferecida, no âmbito do SUS, predominantemente pelo setor privado e pelos hospitais universitários. O financiamento das ações de saúde é típico do modero liberal, com a participação mista tanto do setor público quando do setor privado (Ugá, Porto, \& Piola, 2014). No entanto, o Estado ainda é o maior financiador tanto da atenção básica quanto da atenção de alta complexidade nos hospitais de referência, atuando em ambas as pontas do cuidado à saúde. A quantidade de recursos públicos investidos no setor privado é maior que os gastos públicos diretos em atenção de alta complexidade no Brasil, especialmente em regiões socioeconomicamente mais pobres e com piores condições sanitárias. Esse é o caso das regiões Norte e Nordeste, onde os beneficiários de planos de saúde privada fizeram uso crescente dos serviços ofertados por estabelecimentos credenciados ou financiados pelo SUS (Porto, Ugá, \& Moreira, 2011). Este artigo contribui com uma descrição e reflexão sobre o fornecimento de serviços por esses estabelecimentos (credenciados e/ou contratados pelo SUS), principalmente no que diz respeito à atenção de alta complexidade para condições cardiovasculares.

Até 2007, o financiamento da atenção especializada consistia no pagamento direto ao prestador (hospital) para cada procedimento prestado (Solla \& Chioro, 2014). No entanto, a Portaria GM/MS n. 204, de 29 de janeiro de 2007, estabeleceu que os recursos federais seriam transferidos em uma conta única para os estados, Distrito Federal e municípios, na forma de blocos de financiamento, cada qual constituído por componentes específicos (Portaria GM/MS n. 204, 2007).

O bloco de atenção de média e alta complexidade ambulatorial e hospitalar possui dois componentes: Limite Financeiro da Média e Alta Complexidade Ambulatorial e Hospitalar (MAC), e Fundo de Ações Estratégicas e Compensação (FAEC). No primeiro componente, os recursos federais são transferidos do Fundo Nacional de Saúde aos Fundos de Saúde dos estados, Distrito Federal e municípios, conforme a Programação Pactuada e Integrada (PPI). Os recursos alocados a esse componente passam a incorporar ao fundo do respectivo ente e transferidos regularmente. O segundo componente, o FAEC, foi criado inicialmente para custear os procedimentos de alto custo e de alta complexidade e, depois de gerada uma série histórica, seria incorporado para transferências regulares ao Limite MAC. Atualmente, todos os procedimentos de alta complexidade da área da cardiologia ofertados pelo SUS são pagos por recursos advindos do Limite Financeiro MAC.

No entanto, apesar da criação dos tetos financeiros de MAC e FAEC para a média e alta complexidade, esses componentes não foram suficientes para modificar satisfatoriamente a oferta, no SUS, de serviços públicos para a atenção de média e de alta complexidade. Dessa forma, a lógica de pagamento por 
procedimento permanece, mesmo que indiretamente, vigente no financiamento da saúde pública pelo SUS (Solla \& Chioro, 2014). Nesse sentido, este artigo contribui com uma descrição do fornecimento de serviços pelo setor público ou por estabelecimentos credenciados e/ou contratados pelo SUS, principalmente no que tange à atenção de alta complexidade para condições cardiovasculares.

\section{MÉTODO}

O presente estudo foca na prestação de serviços de saúde tomando por base a dimensão organizacional do Modelo de Giovanella e Fleury (1995). Discute a oferta como uma forma de garantir acesso dentro dos limites geográficos de cada território social (Assis \& Jesus, 2012; Thiede \& McIntyre, 2008; Travassos \& Martins, 2004).

Para avaliar a relação público-privada para serviços de saúde fornecidos ao SUS, a coleta de dados concentrou-se no tipo de estabelecimento de saúde e no tipo e número de procedimentos cardiovasculares realizados. A coleta dos dados foi feita por meio do DATASUS (Departamento de Informática do SUS), que é responsável pelos sistemas de informação em saúde pública no país e disponibiliza dados de 2008 em diante. No presente caso, foram coletados dados secundários para um período de sete anos, compreendido de 2008 a 2014. Esses dados compreenderam o número de leitos hospitalares disponíveis para a realização de procedimentos cardiovasculares autorizados pelo Ministério da Saúde. Utilizou-se estatística descritiva para analisar os dados. A pesquisa não foi submetida a um Comitê de Ética em Pesquisa por fazer uso exclusivo de dados secundários e de domínio público.

O Tipo de Prestador foi uma variável considerada no levantamento dos hospitais que fornecem atenção de alta complexidade para doenças cardiovasculares. Consideraram-se: 1) estabelecimentos públicos (nos níveis da União, dos estados e dos municípios; e 2) estabelecimentos privados, subdivididos em a) organizações sem fins lucrativos, b) organizações filantrópicas e c) organizações com fins lucrativos. ${ }^{2}$

Utilizou-se o Sistema de Informações Hospitalares (SIH / DATASUS) para obter as seguintes variáveis: 1) número de procedimentos e 2) valores aprovados para os serviços hospitalares, ou seja, quanto o sistema público pagou ao hospital que prestou dado serviço de saúde. A análise enfocou nos procedimentos cirúrgicos para atenção de alta complexidade relativa a condições cardiovasculares. Compararam-se a quantidade de procedimentos cirúrgicos realizados e os valores pagos a cada tipo de prestador (i.e., estabelecimento público ou privado) a fim de avaliar as diferenças no financiamento e na prestação de atenção de alta complexidade para condições cardiovasculares.

\section{RESULTADOS E DISCUSSÃO}

A Tabela 1 mostra o número de hospitais financiados pelo SUS e o número de leitos para cirurgia cardiovascular por região e por estado em 2014. Ao todo, 277 hospitais prestaram 697 serviços cirúrgicos nesse período. Observa-se que a região Sudeste concentra praticamente a metade dos hospitais $(\mathrm{N}=125 ; 45 \%)$ e dos serviços $(\mathrm{N}=294 ; 42 \%)$ no país, enquanto a região Centro-Oeste é a que apresenta os menores números.

\footnotetext{
${ }^{2}$ Limitou-se a coleta de dados às instituições prestadoras de serviços para o SUS habilitadas pelo Ministério da Saúde conforme a Portaria SAS/MS no 210/2004.
} 
RAP | Relação público-privada na política brasileira de atenção cardiovascular de alta complexidade

\section{TABELA 1 HOSPITAIS HABILITADOS PELO SUS EM ACC E LEITOS CIRÚRGICOS DE CARDIOLOGIA POR REGIÃO E ESTADO (2014)}

\begin{tabular}{|c|c|c|c|c|c|c|c|c|}
\hline & & & & & tos Cirúrgicc & jara Pro & lemas Cardio & asculares \\
\hline & & & de Hospitais & & )isponível & & SUS & \\
\hline Região & Estado & N & $\begin{array}{c}\text { \%Região / } \\
\text { \% Brasil }\end{array}$ & N & $\begin{array}{c}\text { \%Região / } \\
\text { \% Brasil }\end{array}$ & N & $\begin{array}{c}\text { \%Região / } \\
\text { \% Brasil }\end{array}$ & $\begin{array}{c}\text { \% SUS/ } \\
\text { Total Disponível }\end{array}$ \\
\hline & DF & 2 & 9,0 & 134 & 29,7 & 50 & 20,4 & 37,3 \\
\hline Centro_Oecte (C) & GO & 11 & 50,0 & 188 & 41,6 & 117 & 47,7 & 62,2 \\
\hline centro-Ueste (cu) & MS & 4 & 18,1 & 56 & 12,4 & 29 & 11,8 & 51,7 \\
\hline & MT & 5 & 22,7 & 73 & 16,1 & 49 & 20,0 & 67,1 \\
\hline Total CO & & 22 & $100 / / 7,9$ & 451 & $100 / / 8,1$ & 245 & $100 / / 7,6$ & 54,3 \\
\hline & $A L$ & 3 & 5,3 & 73 & 6,7 & 37 & 5,7 & 50,6 \\
\hline & $\mathrm{BA}$ & 13 & 23,2 & 276 & 25,5 & 134 & 20,7 & 48,5 \\
\hline & CE & 14 & 25,0 & 188 & 17,3 & 134 & 20,7 & 71,2 \\
\hline & MA & 2 & 3,5 & 39 & 3,6 & 22 & 3,4 & 56,4 \\
\hline Nordeste (NE) & PB & 2 & 3,5 & 55 & 5,0 & 44 & 6,8 & 80,0 \\
\hline & PE & 9 & 16,0 & 248 & 22,9 & 173 & 26,7 & 69,7 \\
\hline & $\mathrm{Pl}$ & 5 & 8,9 & 47 & 4,3 & 35 & 5,4 & 74,4 \\
\hline & $\mathrm{RN}$ & 5 & 8,9 & 110 & 10,1 & 46 & 7,1 & 41,8 \\
\hline & SE & 3 & 5,3 & 45 & 4,1 & 22 & 3,4 & 48,8 \\
\hline Total NE & & 56 & $100 / / 20,2$ & 1081 & $100 / / 19,5$ & 647 & $100 / / 20,2$ & 59,8 \\
\hline & $A C$ & 1 & 7,1 & 3 & 0,6 & 3 & 1,1 & 100 \\
\hline & AM & 4 & 28,5 & 71 & 15,5 & 37 & 14,2 & 52,1 \\
\hline & $A P$ & 1 & 7,1 & 151 & 33,0 & 115 & 44,2 & 76,1 \\
\hline Norte (N) & PA & 4 & 28,5 & 179 & 39,1 & 71 & 27,3 & 39,6 \\
\hline & RO & 1 & 7,1 & 21 & 4,6 & 8 & 3,0 & 38,1 \\
\hline & $\mathrm{RR}$ & 0 & - & 10 & 2,1 & 10 & 3,8 & 100 \\
\hline & TO & 3 & 21,4 & 22 & 4,8 & 16 & 6,1 & 72,7 \\
\hline Total N & & 14 & $100 / / 5,0$ & 457 & $100 / / 8,2$ & 260 & $100 / / 8,1$ & 56,8 \\
\hline & ES & 7 & 5,6 & 80 & 3,3 & 48 & 3,5 & 60,0 \\
\hline Sudecte (SF) & $M G$ & 39 & 31,2 & 541 & 22,6 & 308 & 22,5 & 56,9 \\
\hline sudeste (SE) & RJ & 24 & 19,2 & 641 & 26,8 & 323 & 23,6 & 50,3 \\
\hline & $\mathrm{SP}$ & 55 & 44,0 & 1128 & 47,2 & 686 & 50,2 & 60,8 \\
\hline Total SE & & 125 & $100 / / 45,1$ & 2390 & $100 / / 43,2$ & 1365 & $100 / / 42,6$ & 57,1 \\
\hline & PR & 26 & 43,3 & 403 & 35,1 & 218 & 32,0 & 54,0 \\
\hline Sul (S) & $\mathrm{RS}$ & 22 & 36,6 & 463 & 40,4 & 267 & 39,2 & 57,6 \\
\hline & SC & 12 & 20,0 & 280 & 24,4 & 196 & 28,7 & 70,0 \\
\hline Total S & & 60 & $100 / / 21,6$ & 1146 & $100 / / 20,7$ & 681 & $100 / / 21,2$ & 59,4 \\
\hline TOTAL Brasil & & 277 & 100 & 5525 & 100 & 3198 & 100 & 57,8 \\
\hline
\end{tabular}

Fonte: Elaborada pelos autores.

Nota: Em 2014, não havia hospital credenciado ao SUS em Roraima, mas havia leitos cirúrgicos disponíveis em hospitais não vinculados ao SUS. 
A Tabela 1 mostra que 277 hospitais forneceram leitos cirúrgicos para condições cardiovasculares em 2014, sendo que 60\% deles estavam inclusos no SUS, um percentual representativo da maioria das regiões do país. As regiões Nordeste e Sul foram as que tiveram o maior número de leitos credenciados ao SUS. Apenas seis estados tiveram mais leitos disponíveis no setor público que no privado: Distrito Federal (DF), Bahia (BA), Rio Grande do Norte (RN), Sergipe (SE), Pará (PA) e Rondônia (RO). Em Roraima (RR) e no Acre (AC), 100\% dos leitos cirúrgicos para condições cardiovasculares estavam disponíveis para o SUS. Em ambos esses estados, os hospitais com esse tipo de leito eram públicos.

A Tabela 2 mostra os serviços de alta complexidade para condições cardiovasculares prestados em 2014 pelos hospitais licenciados pelo Ministério da Saúde por localidade (região e estado). A maioria dos serviços hospitalares corresponde a cirurgias cardiovasculares e intervencionistas (CCV/ PCI) $(\mathrm{N}=231 ; 33 \%)$ e cirurgias vasculares (VS) $(\mathrm{N}=207 ; 29 \%)$. Em posição intermediária estão os procedimentos endovasculares extracardíacos (PEE) $(\mathrm{N}=108 ; 15 \%)$ e os serviços de Laboratório de Eletrofisiologia $(\mathrm{N}=85 ; 12 \%)$. A Cirurgia Cardiovascular Pediátrica $(\mathrm{CP})$ é o serviço menos prestado $(\mathrm{N}=67 ; 9,6 \%)$.

\section{TABELA 2 SERVIÇOS DE ACC DOS HOSPITAIS HABILITADOS PELO MS POR REGIÃO E ESTADO (2014)}

\begin{tabular}{|c|c|c|c|c|c|c|c|c|c|c|c|}
\hline \multirow[b]{2}{*}{ Região } & \multirow[b]{2}{*}{ Estado } & \multicolumn{2}{|c|}{ CCV/PCI } & \multicolumn{2}{|c|}{$\mathrm{CP}$} & \multicolumn{2}{|c|}{ CV } & \multicolumn{2}{|c|}{ PEE } & \multicolumn{2}{|c|}{ LE } \\
\hline & & $\mathrm{N}$ & $\%$ & $\mathrm{~N}$ & $\%$ & $\mathrm{~N}$ & $\%$ & $\mathrm{~N}$ & $\%$ & $\mathrm{~N}$ & $\%$ \\
\hline \multirow{4}{*}{ Centro-Oeste (CO) } & DF & 2 & 10,5 & 2 & 40,0 & 2 & 14,2 & 1 & 12,5 & 2 & 22,2 \\
\hline & G0 & 9 & 47,3 & 1 & 20,0 & 5 & 35,7 & 2 & 25,0 & 3 & 33,3 \\
\hline & MS & 3 & 15,7 & 1 & 20,0 & 4 & 28,5 & 2 & 25,0 & 1 & 11,1 \\
\hline & MT & 5 & 26,3 & 1 & 20,0 & 3 & 21,4 & 3 & 37,5 & 3 & 33,3 \\
\hline \multicolumn{2}{|l|}{ Total CO } & 19 & 8,2 & 5 & 7,4 & 14 & 6,7 & 8 & 7,4 & 9 & 10,5 \\
\hline \multirow{9}{*}{ Nordeste (NE) } & $\mathrm{AL}$ & 3 & 6,3 & 1 & 4,7 & 2 & 5,2 & 2 & 9,0 & 3 & 14,2 \\
\hline & BA & 12 & 25,5 & 7 & 33,3 & 12 & 31,5 & 6 & 27,2 & 5 & 23,8 \\
\hline & CE & 10 & 21,2 & 3 & 14,2 & 6 & 15,7 & 3 & 13,6 & 2 & 9,5 \\
\hline & MA & 2 & 4,2 & 2 & 9,5 & 2 & 5,2 & 1 & 4,5 & 1 & 4,7 \\
\hline & PB & 3 & 6,3 & 0 & - & 3 & 7,8 & 1 & 4,5 & 1 & 4,7 \\
\hline & PE & 8 & 17,0 & 3 & 14,2 & 6 & 15,7 & 5 & 22,7 & 5 & 23,8 \\
\hline & $\mathrm{Pl}$ & 3 & 6,3 & 2 & 9,5 & 4 & 10,5 & 2 & 9,0 & 1 & 4,7 \\
\hline & RN & 5 & 10,6 & 1 & 4,7 & 2 & 5,2 & 1 & 4,5 & 2 & 9,5 \\
\hline & SE & 1 & 2,1 & 2 & 9,5 & 1 & 2,6 & 1 & 4,5 & 1 & 4,7 \\
\hline Total NE & & 47 & 20,3 & 21 & 31,3 & 38 & 18,3 & 22 & 20,3 & 21 & 24,7 \\
\hline
\end{tabular}


RAP | Relação público-privada na política brasileira de atenção cardiovascular de alta complexidade

\begin{tabular}{|c|c|c|c|c|c|c|c|c|c|c|c|}
\hline \multirow[b]{2}{*}{ Região } & \multirow[b]{2}{*}{ Estado } & \multicolumn{2}{|c|}{ CCV/PCI } & \multicolumn{2}{|c|}{$\mathrm{CP}$} & \multicolumn{2}{|c|}{ CV } & \multicolumn{2}{|c|}{ PEE } & \multicolumn{2}{|c|}{ LE } \\
\hline & & $\mathrm{N}$ & $\%$ & $\mathrm{~N}$ & $\%$ & $\mathrm{~N}$ & $\%$ & $N$ & $\%$ & $\mathrm{~N}$ & $\%$ \\
\hline \multirow{7}{*}{ Norte (N) } & $A C$ & 1 & 8,3 & 0 & - & 0 & - & 0 & - & 0 & - \\
\hline & AM & 3 & 25,0 & 0 & - & 2 & 20,0 & 1 & 20,0 & 2 & 40,0 \\
\hline & $A P$ & 1 & 8,3 & 0 & - & 0 & - & 0 & - & 0 & - \\
\hline & PA & 4 & 33,3 & 2 & 100 & 4 & 40,0 & 2 & 40,0 & 2 & 40,0 \\
\hline & Ro & 1 & 8,3 & 0 & - & 1 & 10,0 & 1 & 20,0 & 0 & - \\
\hline & $\mathrm{RR}$ & 0 & - & 0 & - & 0 & - & 0 & - & 0 & - \\
\hline & TO & 2 & 16,6 & 0 & - & 3 & 30,0 & 1 & 20,0 & 1 & 20,0 \\
\hline Total N & & 12 & 5,9 & 2 & 2,9 & 10 & 4,8 & 5 & 4,6 & 5 & 5,8 \\
\hline \multirow{4}{*}{ Sudeste (SE) } & ES & 6 & 5,7 & 2 & 7,6 & 6 & 6,5 & 3 & 7,1 & 2 & 6,4 \\
\hline & MG & 32 & 30,7 & 8 & 30,7 & 29 & 31,8 & 16 & 38,1 & 11 & 35,4 \\
\hline & RJ & 20 & 19,2 & 4 & 15,3 & 18 & 19.7 & 6 & 14,2 & 6 & 19,3 \\
\hline & $\mathrm{SP}$ & 46 & 44,2 & 12 & 46,1 & 38 & 41,7 & 17 & 40,4 & 12 & 38,7 \\
\hline \multirow[t]{2}{*}{ Total SE } & & 104 & 45,0 & 26 & 38,8 & 91 & 43,9 & 42 & 38,8 & 31 & 36,4 \\
\hline & PR & 22 & 44,9 & 7 & 53.9 & 25 & 46.3 & 12 & 38,7 & 6 & 31,6 \\
\hline \multirow[t]{2}{*}{ Sul (S) } & RS & 17 & 34,6 & 4 & 30,8 & 20 & 37,0 & 13 & 41,9 & 10 & 52,6 \\
\hline & SC & 10 & 20,4 & 2 & 15,4 & 9 & 16,7 & 6 & 19,4 & 3 & 15,8 \\
\hline Total S & & 49 & 21,2 & 13 & 19,4 & 54 & 26,0 & 31 & 28,7 & 19 & 22,3 \\
\hline TOTAL Brasil & & 231 & 33,0 & 67 & 9,6 & 207 & 29,6 & 108 & 15,4 & 85 & 12,1 \\
\hline
\end{tabular}

Fonte: Elaborada pelos autores.

Nota: $\mathrm{CCV} / \mathrm{PCI}$ = Cirurgia Cardiovascular e Intervencionista; CP = Cirurgia Cardiovascular Pediátrica; CV = Cirurgia Vascular; $\mathrm{PEE}=$ Procedimentos Endovasculares Extracardíacos; LE = Laboratório de Eletrofisiologia.

A Tabela 2 também mostra algumas lacunas no cuidado à saúde, ou seja, alguns estados não dispõem de prestadores de serviços de alta complexidade para condições cardiovasculares ou não oferecem alguns serviços nessa área. A região Norte oferece o menor número de serviços na área, com a seguinte distribuição em seus estados: nenhuma organização que realize cirurgias cardiovasculares em Roraima (RR); um hospital que oferece apenas cirurgias cardiovasculares e intervencionistas em cada um dos estados do Acre (AC) e do Amapá (AP); nenhuma organização que ofereça cirurgias cardiovasculares pediátricas ou laboratórios de eletrofisiologia em Rondônia; e nenhum hospital que ofereça cirurgias cardiovasculares pediátricas no Amazonas (AM) e no Tocantins (TO).

A Tabela 3 mostra que $73 \%(\mathrm{~N}=203)$ dos fornecedores de serviços de alta complexidade para condições cardiovasculares credenciados ao SUS eram do setor privado. Esse número compreendia organizações filantrópicas $(\mathrm{N}=136 ; 67 \%)$, organizações com fins lucrativos $(\mathrm{N}=43 ; 21 \%)$ e organizações sem fins lucrativos $(\mathrm{N}=24 ; 12 \%)$. 


\section{TABELA 3 HOSPITAIS CREDENCIADOS AO SUS QUE PRESTAM ATENÇÃO DE ALTA COMPLEXIDADE PARA CONDIÇÕES CARDIOVASCULARES POR REGIÃO E ESTADO, SEGUNDO TIPO DE PRESTADOR (2014)}

\begin{tabular}{|c|c|c|c|c|c|c|c|c|c|c|c|c|c|}
\hline \multirow[b]{3}{*}{ Região } & \multirow[b]{3}{*}{ Estado } & \multicolumn{8}{|c|}{ Instituição Privada } & & & & \multirow{3}{*}{$\begin{array}{c}\% \\
\text { Público }\end{array}$} \\
\hline & & \multicolumn{2}{|c|}{ Filantrópica } & \multicolumn{2}{|c|}{$\begin{array}{l}\text { Com Fins } \\
\text { Lucrativos }\end{array}$} & \multicolumn{2}{|c|}{$\begin{array}{l}\text { Sem Fins } \\
\text { Lucrativos }\end{array}$} & \multicolumn{2}{|c|}{$\begin{array}{l}\text { Total de } \\
\text { Instituições } \\
\text { Privadas }\end{array}$} & \multicolumn{2}{|c|}{$\begin{array}{c}\text { Total de } \\
\text { Organizações } \\
\text { Públicas }\end{array}$} & \multirow{2}{*}{$\begin{array}{c}\% \\
\text { Privado }\end{array}$} & \\
\hline & & $\mathrm{N}$ & $\%$ & $\mathrm{~N}$ & $\%$ & $\mathrm{~N}$ & $\%$ & $N$ & $\%$ & $\mathrm{~N}$ & $\%$ & & \\
\hline \multirow{4}{*}{$\begin{array}{l}\text { Centro-Oeste } \\
\text { (CO) }\end{array}$} & DF & 1 & 8,3 & 0 & - & 0 & - & 1 & 5,8 & 1 & 20,0 & 50,0 & 50,0 \\
\hline & G0 & 3 & 25 & 3 & 100 & 2 & 100 & 8 & 47,0 & 3 & 60,0 & 72,7 & 27,2 \\
\hline & MS & 3 & 25 & 0 & - & 0 & - & 3 & 17,6 & 1 & 20,0 & 75,0 & 25,0 \\
\hline & MT & 4 & 33,3 & 0 & - & 0 & - & 4 & 23,5 & 1 & 20,0 & 80,0 & 20,0 \\
\hline \multirow[t]{5}{*}{ Total CO } & & 11 & 8 & 3 & 6,9 & 2 & 8,3 & 16 & 8,3 & 6 & 8,1 & 72,7 & 27,2 \\
\hline & $A L$ & 2 & 6,4 & 0 & - & 0 & - & 2 & 4,3 & 1 & 10,0 & 66,6 & 33,3 \\
\hline & BA & 9 & 29 & 2 & 15,3 & 0 & - & 11 & 23,9 & 2 & 20,0 & 84,6 & 15,3 \\
\hline & CE & 5 & 16,1 & 3 & 23,0 & 1 & 50,0 & 9 & 19,5 & 5 & 50,0 & 64,2 & 35,7 \\
\hline & MA & 1 & 3,2 & 1 & 7,6 & 0 & - & 2 & 4,3 & 0 & & 100 & 0,0 \\
\hline \multirow[t]{5}{*}{ Nordeste (NE) } & PB & 2 & 6,4 & 1 & 7,6 & 0 & - & 3 & 6,5 & 0 & & 100 & 0,0 \\
\hline & PE & 6 & 19,3 & 3 & 23,0 & 0 & & 9 & 19,5 & 0 & & 100 & 0,0 \\
\hline & PI & 2 & 6,4 & 1 & 7,6 & 0 & & 3 & 6,5 & 1 & 10,0 & 75,0 & 25,0 \\
\hline & RN & 2 & 6,6 & 1 & 7,6 & 1 & 50,0 & 4 & 8,8 & 1 & & 80,0 & 20,0 \\
\hline & SE & 1 & 3,2 & 1 & 7,6 & 0 & & 2 & 4,3 & 1 & 10,0 & 66,6 & 33,3 \\
\hline \multirow[t]{3}{*}{ Total NE } & & 30 & 22 & 13 & 30,2 & 2 & 8,3 & 45 & 22,0 & 11 & 14,8 & 80,3 & 19,6 \\
\hline & $A C$ & 0 & - & 0 & - & 1 & 25,0 & 1 & 9,0 & 0 & & 100 & 0,0 \\
\hline & AM & 3 & 60 & 0 & - & 1 & 25,0 & 4 & 36,3 & 0 & & 100 & 0,0 \\
\hline \multirow{4}{*}{ Norte (N) } & AP & 0 & - & 0 & - & 1 & 25,0 & 1 & 9,0 & 0 & & 100 & 0,0 \\
\hline & PA & 1 & 20 & 2 & 100 & 1 & 25,0 & 4 & 36,3 & 0 & & 100 & 0,0 \\
\hline & RO & 0 & - & 0 & - & 0 & & 0 & & 1 & 33,3 & 0,0 & 100 \\
\hline & TO & 1 & 20 & 0 & - & 1 & 25,0 & 2 & 18,1 & 1 & 33,3 & 66,6 & 33,3 \\
\hline \multirow[t]{2}{*}{ Total N } & & 5 & 3,6 & 2 & 4,6 & 5 & 20,8 & 12 & 5,8 & 2 & 2,7 & 85,7 & 14,2 \\
\hline & ES & 1 & 1,7 & 0 & & 1 & 8,3 & 2 & 2,3 & 4 & 10,5 & 33,3 & 66,6 \\
\hline \multirow{3}{*}{ Sudeste (SE) } & $M G$ & 21 & 37,5 & 7 & 36,8 & 3 & 25,0 & 31 & 35,6 & 8 & 21,0 & 79,4 & 20,5 \\
\hline & RJ & 11 & 19,6 & 5 & 26,3 & 1 & 8,3 & 17 & 19,5 & 7 & 18,4 & 70,8 & 29,1 \\
\hline & $\mathrm{SP}$ & 23 & 41,0 & 7 & 36,8 & 7 & 58,3 & 37 & 42,5 & 19 & 50,0 & 66,0 & 33,9 \\
\hline \multirow[t]{2}{*}{ Total SE } & & 56 & 41,1 & 19 & 44,1 & 12 & 50 & 87 & 42,6 & 38 & 51,3 & 69,6 & 30,4 \\
\hline & PR & 13 & 38,2 & 3 & 50,0 & 3 & 100 & 19 & 44,1 & 7 & 41,1 & 73,0 & 26,9 \\
\hline \multirow[t]{2}{*}{ Sul (S) } & $\mathrm{RS}$ & 13 & 38,2 & 2 & 33,3 & 0 & & 15 & 34,8 & 7 & 41,1 & 68,1 & 31,8 \\
\hline & SC & 8 & 23,5 & 1 & 16,6 & 0 & & 9 & 20,9 & 3 & 17,6 & 75,0 & 25,0 \\
\hline Total S & & 34 & 25,0 & 6 & 13,9 & 3 & 12,5 & 43 & 21,0 & 17 & 22,9 & 71,6 & 28,3 \\
\hline TOTAL Brasil & & 136 & 67,0 & 43 & 21,1 & 24 & 11,8 & 203 & 100 & 74 & 100 & 73,2 & 26,7 \\
\hline
\end{tabular}

Fonte: CNES (2014). 
A Tabela 3 também mostra que a porcentagem de organizações privadas prestadoras de atenção de alta complexidade varia de 69\%, no Sudeste, a $86 \%$, no Norte, claramente evidenciando o predomínio do setor privado na prestação desses serviços em todo o Brasil. Em alguns estados, todos os hospitais que fornecem tais serviços pertencem ao setor privado: Maranhão (MA), Paraíba (PB) e Pernambuco (PE), no Nordeste; Acre (AC), Amazonas (AM), Amapá (AP) e Pará (PA), no Norte.

A Tabela 4 exibe o número de habilitações pelo SUS por tipo de prestador de 2008 a 2014. O setor privado tem o maior número de aumento (mais de 28\%), de 158 hospitais em 2008 para 203 em 2014, enquanto o setor público teve um aumento de $12 \%$, passando de 66 para 74 hospitais no mesmo período. As organizações filantrópicas são as que tiveram a maior taxa de aumento $(22,5 \%)$. Em suma, há uma clara tendência de habilitação de organizações privadas em detrimento de estabelecimentos públicos. Em 2014, a habilitação de hospitais privado superou o número de hospitais públicos em quase três vezes.

\section{TABELA 4 HOSPITAIS HABILITADOS EM ACC POR TIPO DE PRESTADOR (2008-2014)}

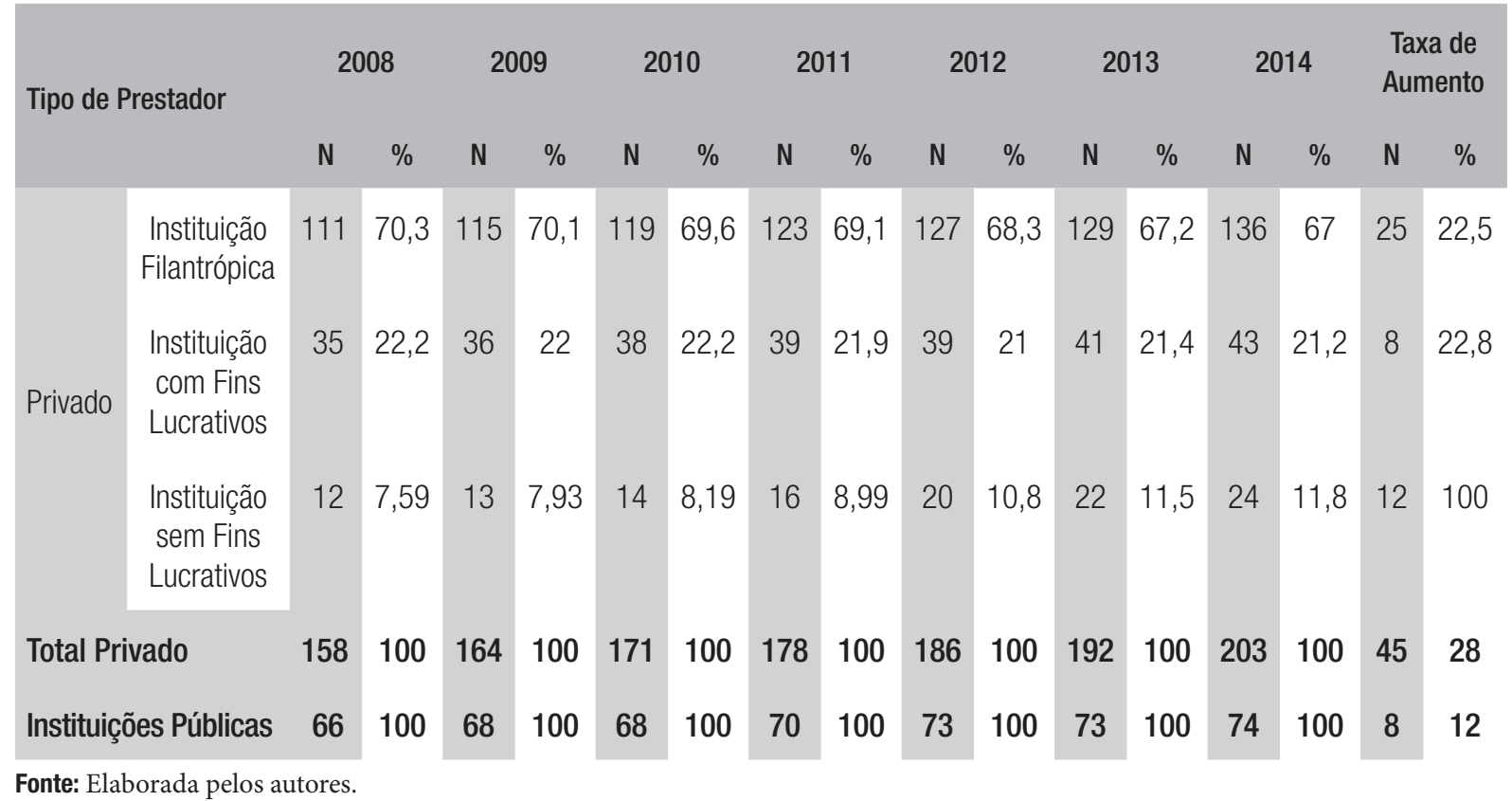

A Tabela 5 segmenta por estabelecimentos públicos e privados o número de procedimentos de alta complexidade e os gastos financeiros, em reais, relativos às condições cardiovasculares. Os hospitais privados são responsáveis por 144.584/ano (71\%) procedimentos cirúrgicos cardiovasculares, consumindo cerca de $72 \%$ ( $\mathrm{R}$ 972.503.672/ano) dos recursos disponíveis para esse tipo de atenção. Por sua vez, o setor público realiza 29\% (59.020/ano) dos procedimentos, recebendo 27\% (368.977.148) dos recursos. 


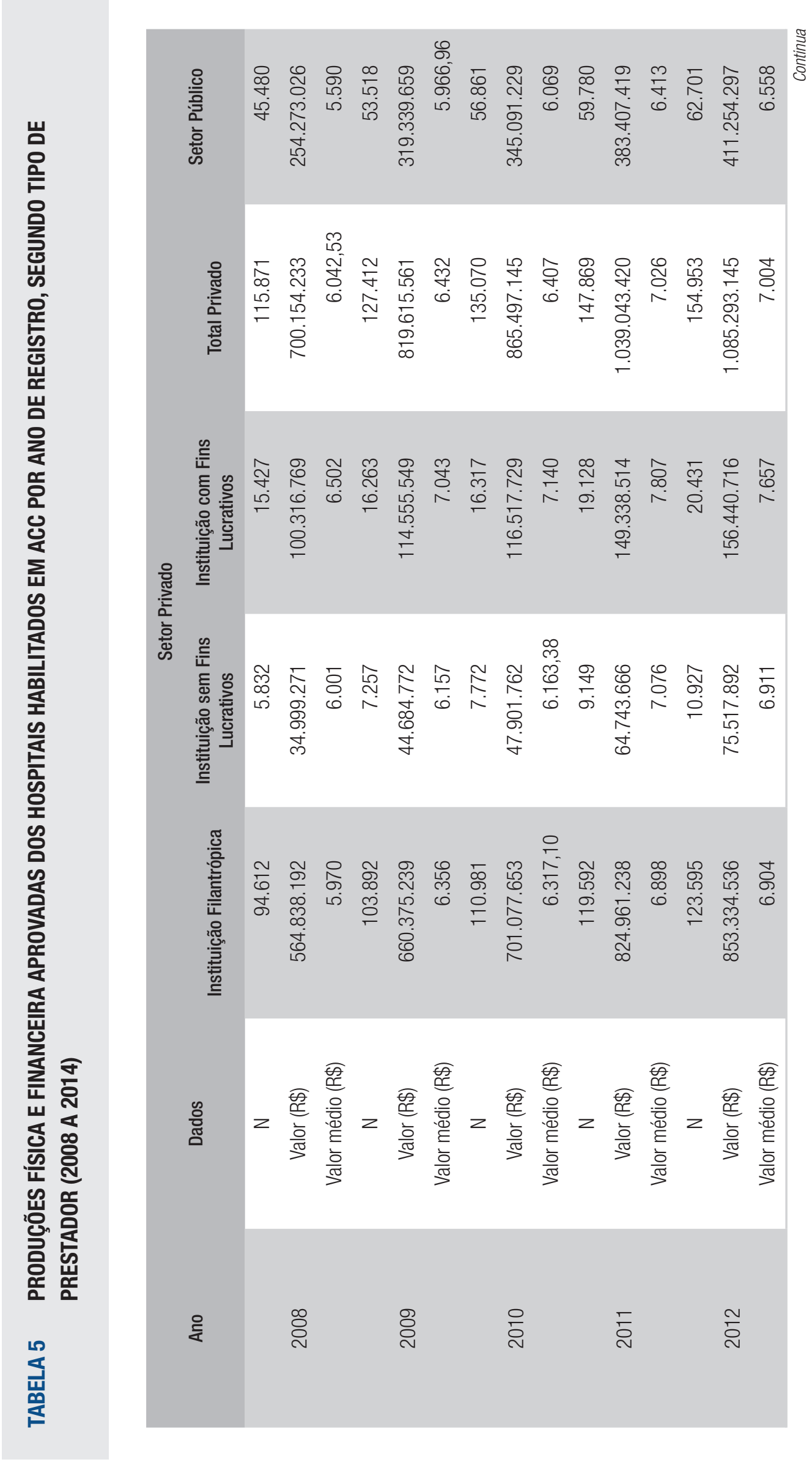

REVISta de AdMinistração PÚblica | Rio de Janeiro 53(4):753-768, jul. - ago. 2019 


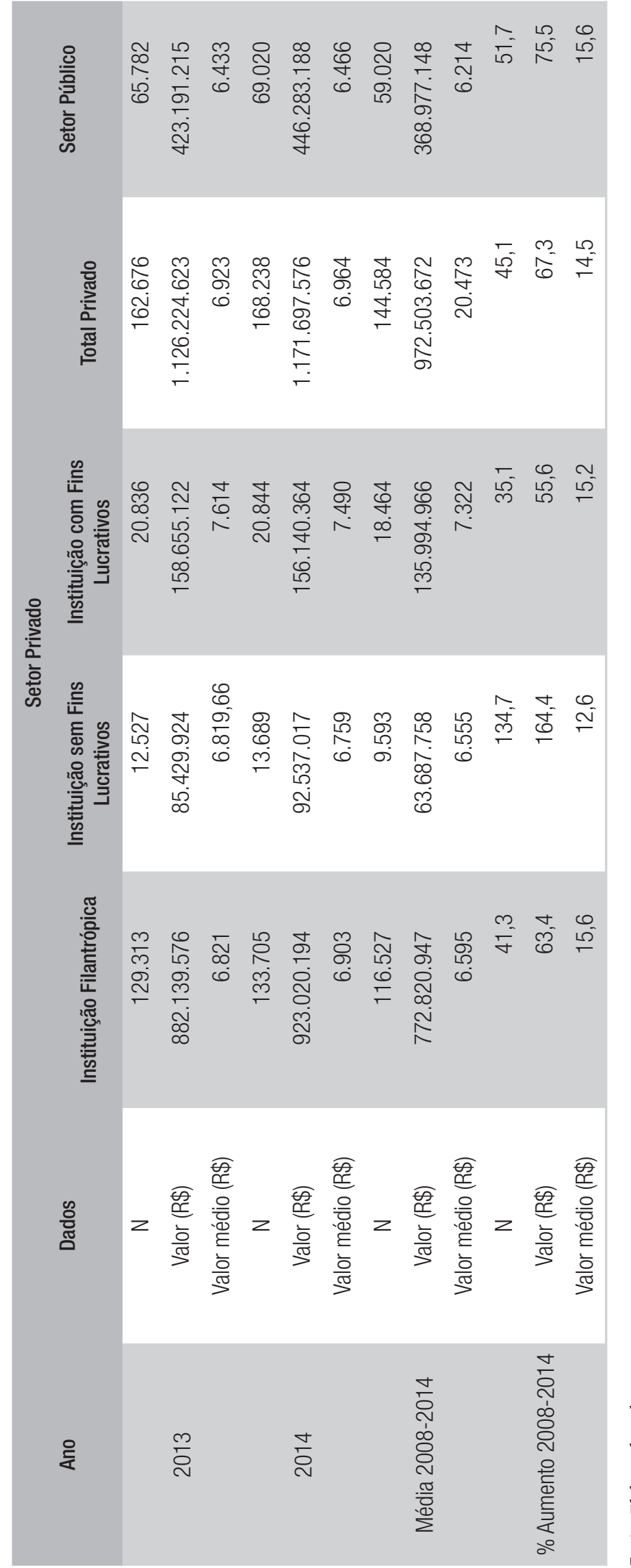


Considerando o número de procedimentos cirúrgicos realizados de 2008 a 2014 (cf. Tabela 5), a tendência na prestação de atenção de alta complexidade para condições cardiovasculares no Brasil aponta para um maior crescimento entre os estabelecimentos públicas (52\%; de 45.480 em 2008 para 69.020 em 2014) e um menor crescimento entre os privados (45\%; de 115.871 em 2008 para 168.238 em 2014). Também se verifica uma tendência similar nos gastos financeiros (cf. Tabela 5): um maior aumento, de aproximadamente 75\%, no setor público (de R $\$ 254.273 .026,00$ em 2018 para R\$ 446.283.188 em 2014); e um menor aumento, de aproximadamente 67\%, no setor privado (de $\mathrm{R} \$$ 700.154.233 em 2008 para 1.171.697.576 em 2014).

Contudo, os estabelecimentos privados são responsáveis pela maioria da atenção de alta complexidade para condições cardiovasculares no Brasil e prestam os procedimentos mais onerosos. Isso sugere que a atenção de alta complexidade para condições cardiovasculares é atraente para o setor privado, em especial no que diz respeito aos procedimentos mais caros: os hospitais privados e os públicos oferecem procedimentos que custam, respectivamente cerca de $\mathrm{R} \$ 21.000$ e R 6.300 (cf. Tabela 5). Portanto, os custos de saúde vêm crescendo excessivamente e têm gerado uma lógica de financiamento que favorece os serviços mais sofisticados e caros, que fazem uso intensivo de tecnologia, em detrimento daqueles mais simples e menos dispendiosos, por mais que a atenção básica seja mais universal em termos de oferta.

Tais resultados evidenciam que a rede de atenção de alta complexidade para condições cardiovasculares é bastante extensa e distribuída de forma desproporcional em todo o país. Identificam-se concentrações e lacunas no número de hospitais e no número de leitos cirúrgicos cardiovasculares por localidade (regiões e estados). Enquanto a região Sul apresenta a maior concentração de estabelecimentos e ofertado o maior número de serviços cardiovasculares (quase a metade de todo o país), a região norte é responsável por menos de $6 \%$ dos hospitais e dos serviços. Isso pode ser explicado pelo fato de que essas regiões são aquelas com, respectivamente, o maior e o menor resultado para densidade populacional e índice de desenvolvimento humano (Instituto Brasileiro de Geografia e Estatística [IBGE], 2014). O risco de tais lacunas está na falta de atendimento de saúde para pacientes com necessidade de cirurgia cardiovascular. Por exemplo, diversos estudos (p. ex., Oram, Buckenham, Roake, \& Lewis, 2008; Turnbull et al., 2000) mostram que diagnóstico, exame e tratamento tardios de pacientes com arterial obstrutiva periférica aumentam as complicações e a mortalidade.

Embora o SUS tenha desenvolvido suas estratégias de financiamento para atenção de média e alta complexidade a partir de um modelo de blocos de financiamento e permita que os estados e municípios aloquem livremente seus recursos, a prática tem sido de financiamento indireto, com os pagamentos sendo realizados por procedimentos. Isso corrobora Mendes (2011) e Solla e Chioro (2014), que apontam para a sobreposição dos interesses privados em detrimento das necessidades reais de um sistema de saúde universal. O SUS admite serviços privados por meio de contratos e acordos, o que reduz sua capacidade de condicionar o fornecimento às prioridades e necessidades de saúde da população, pois a dinâmica do mercado segue a lógica da oferta, e não da demanda. Assim, os preços podem ser arbitrários, e o fornecimento pode se basear exclusivamente naqueles procedimentos que o prestador de serviços privados decide ofertar. 


\section{CONSIDERAÇÕES FINAIS}

O SUS foi estabelecido em 1988 como um direito de todos os cidadãos (i.e., um sistema universal) e um dever do Estado a fim de oferecer saúde gratuita e integral em todos os níveis de complexidade. A participação do setor privado no SUS foi permitida, em tese, como um complemento para a prestação de serviços de saúde sob financiamento público. Contudo, os hospitais privados têm cada vez mais fornecido atenção de média e alta complexidade, o que tem aumentado a alocação de recursos públicos nos estabelecimentos privados.

O presente estudo especificamente demonstra que a participação privada na atenção de alta complexidade para condições cardiovasculares não segue uma lógica de complementaridade no país, com um predomínio crescente do setor privado. Os estabelecimentos privados têm recebido pagamentos maiores que os públicos para a atenção especializada, uma vez que fornecem ao Estado serviços mais dispendiosos. A estrutura pública da atenção especializada no Brasil é insuficiente para cumprir os princípios de universalidade e integralidade do SUS (Solla \& Chioro, 2014). Isso parece ter levado a um aumento na participação do setor privado no fornecimento de atenção especializada para condições cardiovasculares.

Como discutido por Santos (2010), tratar a relação público-privado como dois polos concorrentes parece não ajudar na melhoria da gestão da atenção de alta complexidade no Brasil. Pelo contrário, esses polos deveriam ser vistos como parceiros na implementação dos princípios do SUS. Portanto, como proposto por Mendes (2011), é necessário conceber estratégias de gestão que busquem organizar a rede de atenção de média e alta complexidade em conjunto com a atenção primária nos estabelecimentos de saúde em diferentes localidades. Guerra e Gonçalves (2014) defendem que os leitos hospitalares para atenção de alta complexidade deveriam ser disponíveis a qualquer indivíduo, independentemente do município ou estado em que resida. Outro passo importante é aumentar o controle regulatório sobre o fornecimento e o pagamento de procedimentos de alta complexidade a fim de evitar contratos e acordos ilegais, unilaterais e danosos. Além disso, a lógica de habilitação do SUS, baseada no fornecimento de serviços pelo setor privado, também é problemática para o sistema. Logo, o SUS deveria optar por uma lógica de demanda requerida, e não por uma lógica de oferta determinada pelo setor privado.

Uma possibilidade de se racionalizar o modo de financiamento da atenção de alta complexidade é adotando a lógica capitalista de alocação de recursos. Essa lógica considera: 1) a segmentação dos serviços de saúde pelo nível de atenção; 2) as demandas de saúde como representativas das condições de saúde da população; 3) a estruturação dos hospitais que fornecem atenção de alta complexidade através de redes de atenção integrada (Guerra \& Gonçalves, 2014).

Dada a importância de se conhecer melhor a relação público-privada na atenção de média e alta complexidade no sistema público de saúde brasileiro, sugere-se que estudos futuros investiguem o impacto que o desempenho dos hospitais que fornecem atenção de alta complexidade tem na recuperação dos pacientes e no uso dos recursos que são repassados pela administração pública. Esta análise poderia ser inclusa como um critério da PNANAC, auxiliando o Estado a direcionar organizações hospitalares para lugares onde haja reduzida disponibilidade de leitos. Esta e outras diretrizes compõem o atual documento da OECD (2010) sobre governança em saúde. 


\section{REFERÊNCIAS}

Alexandrino, M.; Paulo, V. (2000). Direito Administrativo descomplicado (21a ed.). Rio de Janeiro: Forense, São Paulo, SP: Método.

Arrow, K. (1963). Uncertainty and the welfare economics of medical care. American Economic Review, 53(5), 941-973.

Assis, M. M. A., \& Jesus, W. L. A. (2012). Acesso aos serviços de saúde: abordagens, conceitos, políticas e modelo de análise. Ciências \& Saúde Coletiva, 17(11), 2865-2875.

Barata, L. R. B., Tanaka, O. Y., \& Mendes, J. D. V. (2014). Por um processo de descentralização que consolide os princípios do Sistema Único de Saúde. Epidemiologia e Serviços de Saúde, 13(1), 15-24.

Gadelha, C. A. G. (2012). A saúde no Brasil em 2030: diretrizes para a prospecção estratégica do sistema de saúde brasileiro. Rio de Janeiro, RJ: Fiocruz/ Ipea/Ministério da Saúde/Secretaria de Assuntos Estratégicos da Presidência da República.

Gadelha, C. A. G., Quental, C., \& Fialho, B. C. (2003). Saúde e inovação: uma abordagem sistêmica das indústrias da saúde. Cadernos de Saúde Pública, 19(1), 47-59.

Giovanella, L., \& Fleury, S. (1995). Universalidade da Atenção à Saúde: acesso como categoria de análise. In C. Eibenschutz (Org.), Política de Saúde: o público e o privado (pp. 177-198). Rio de Janeiro, RJ: Fiocruz.

Grossman, M. (1972). On the concept of health capita and the demand for health. Journal of Political Economy, 80(2), 223-255.

Guerra, M., \& Gonçalves, A. O. (2014). Model of allocating public financial resources to Brazilian hospital organization that provide highly complex healthcare services. Open Medicine Journal, 1(78), 78-89.

Instituto Brasileiro de Geografia e Estatística. (2014). Estimativas da população residente no Brasil e Unidades da Federação com data de referência em $1^{\circ}$ de julho de 2014. Rio de Janeiro, RJ: IBGE. Recuperado de ftp://ftp.ibge.gov.br/Estimativas_de_Populacao/ Estimativas_2014/estimativa_dou_201 4.pdf

Malta, D. C., Moura, L., Prado, R. R., Escalante, J. C., Schimidt, M. I., \& Duncan, B. B. (2014). Mortalidade por doenças crônicas não transmissíveis no Brasil e suas regiões, 2000 a 2011. Epidemiologia, Serviço e Saúde, 23(4), 599-608.
Marchildon, G. P. (2015). The crisis of regionalization. Health Manage Forum, 28(6), 236-238.

Mello, G. A., Pereira, A. P. C. M., Uchimura, L. Y. T., Iozzi, F. L., Demarzo, M. M. P., \& Viana, A. L. d'A. (2017). O processo de regionalização do SUS: revisão sistemática. Ciência \& Saúde Coletiva, 22(4), 1291-1310.

Mendes, E. V. (2011). As redes de atenção à saúde. Brasília: Organização Pan-Americana da Saúde.

Oliver, K., Lorenc, T., \& Innvær, S. (2014). New directions in evidence-based policy research: a critical analysis of the literature. Health Research Policy and Systems, 12(34), 1-11.

Oram, B. J., Buckenham, T. M., Roake, J. A., \& Lewis, D. R. (2008). Treatment delays for patients exiting an aortic aneurysm surveillance programme. $A N Z$ Journal of Surgery, 78(3), 148-150.

Pinto, V. C., Jr., Fraga, M. N. O., \& Freitas, S. M. (2012). Análise das portarias que regulamentam a Política Nacional de Atenção Cardiovascular de Alta Complexidade. Revista Brasileira de Cirurgia Cardiovascular, 27(3), 463-468.

Portaria GM/MS n. 204, de 29 de janeiro de 2007. (2007). Regulamenta o financiamento e a transferência dos recursos federais para as ações e os serviços de saúde, na forma de blocos de financiamento, com o respectivo monitoramento e controle. Recuperado de http://bvsms.saude.gov.br/bvs/saudelegis/gm/2007/ prt0204_29_01_2007_comp.html

Portaria GM/MS n. 4.279, de 30 de dezembro de 2010. (2010). Estabelece diretrizes para a organização da Rede de Atenção à Saúde no âmbito do Sistema Único de Saúde (SUS). Recuperado de http:// bvsms.saude.gov.br/bvs/saudelegis/gm/2010/ prt4279_30_12_2010.html

Portaria SAS/MS n. 433, de 15 de maio de 2012. (2012). Suspende os parâmetros populacionais para habilitação em Média ou Alta Complexidade das áreas de Cardiologia, Oftalmologia, Nefrologia e Neurocirurgia. Recuperado de http://bvsms.saude.gov. br/bvs/saudelegis/sas/2012/prt0433_15_05_2012.html

Porto, S. M., Ugá, M. A. D., \& Moreira, R. S. (2011). An analysis of use of the health services by financing system: Brazil 1998-2008. Ciência \& Saúde Coletiva, 16(9), 3795-3806.

Ribeiro, J. M., Moreira, M. R., Ouverney, A. M., \& Silva, C. M. F. P. (2017). Políticas de saúde e lacunas 
federativas no Brasil: uma análise da capacidade regional de provisão de serviços. Ciência \& Saúde Coletiva, 22(4), 1031-1044.

Ribeiro, J. M., \& Alcoforado, F. (2016). Mecanismos de governança e o desenho institucional da Secretaria de Saúde do Município do Rio de Janeiro (RJ). Ciência \& Saúde Coletiva, 21(5), 1339-1349.

Romano, C. M. C., \& Scatena, J. H. G. (2014). A relação público - privada no SUS: um olhar sobre a assistência ambulatorial de média e alta complexidade na região de saúde da Baixada Cuiabana em Mato Grosso. Revista de Administração Pública, 48(2), 439-458.

Santos, L. Administração pública e a gestão da saúde. (2010). In: Santos, N. R., \& Amarante, P. D.C. (Org.), Gestão pública e relação público privado na saúde (pp. 68-86). Rio de Janeiro, RJ: Cebes.

Servo, L. M. S., Piola, S. F., Paiva, A. B., \& Ribeiro, J. A. (2011). Financiamento e gasto público de saúde: histórico e tendências. In: C. Melamed, \& S. F. Piola
(Orgs.), Políticas Públicas e Financiamento Federal do Sistema Único de Saúde (pp. 85-108). Brasília, DF: Ipea.

Solla, J. J. S. P., \& Chioro, A. A. R. (2014). Atenção Ambulatorial Especializada. In L. Giovanella (Org.), Políticas e Sistemas de Saúde no Brasil (2a ed., pp. 547-576). Rio de Janeiro, RJ: Fiocruz.

Travassos, C., \& Martins, M. (2004). Uma revisão sobre os conceitos de acesso e utilização de serviços de saúde. Cadernos de Saúde Pública, 20(Sup. 2), S190-S198.

Turnbull, R. G., Taylor, D. C., Hsiang, Y. N., Salvian, A. J., Nanji, S., O'Hanley, G., Doyle, D. L., \& Fry, P. D. (2000). Assessment of patient waiting times for vascular surgery. Canadian Journal of Surgery, 43(2), 105-111.

Ugá, M. A. D., Porto, S. M., \& Piola, S. F. (2014). Financiamento e Alocação de Recursos em Saúde no Brasil. In: L. Giovanella (Org.), Politicas e Sistemas de Saúde no Brasil (2a ed., pp. 395-426). Rio de Janeiro, RJ: Fiocruz.

\section{Petter Ricardo de Oliveira}

https://orcid.org/0000-0002-1224-4286

Mestre em Saúde Coletiva pela Universidade de Brasília (UnB).E-mail: petterpro@yahoo.com.br

\section{Mariana Guerra}

https://orcid.org/0000-0001-5234-5172

Doutora em Administração pela Universidade de Brasília (UnB); Professora Adjunta do Departamento de Ciências Contábeis e Atuariais e do Programa de Pós-Graduação em Contabilidade da Universidade de Brasília (PPGCont/UnB). E-mail: profamarianaguerra@gmail.com

\section{Adalmir de Oliveira Gomes}

https://orcid.org/0000-0001-9000-3296

Doutor e Mestre em Administração pela Universidade de Brasília (UnB); Professor Adjunto do Departamento de Administração e do Programa de Pós-Graduação em Administração da Universidade de Brasília (PPGA/ UnB). E-mail: adalmirdeoliveira@gmail.com

\section{Aiane Luiz Martins}

https://orcid.org/0000-0003-3082-6180

Mestra em Ciências Contábeis pelo Programa de Pós-Graduação em Ciências Contábeis da Universidade de Brasília (UnB); Professora no Instituto Euro-Americano de Educação, Ciência e Tecnologia (EUROAM). E-mail: aiane.martins@unieuro.com.br 